\title{
Ultrashort-Term Power Fluctuation Forecasting Based on the Prediction of the Shipborne Panel Tilt Angle
}

\author{
Xiuyan Peng ${ }^{1}$, Luo Zhao ${ }^{1, *}$, Bo Wang ${ }^{1}$ and Biao Zhang ${ }^{2}$ (-) \\ 1 College of Automation, Harbin Engineering University, Harbin 150001, China; \\ pengxiuyan@hrbeu.edu.cn (X.P.); excalibur@hrbeu.edu.cn (B.W.) \\ 2 School of Automation, Harbin University of Science and Technology, Harbin 150080, China; \\ zhangbiaohlj@163.com \\ * Correspondence: zhaoluopt@hrbeu.edu.cn; Tel.: +86-188-4559-5805
}

Received: 20 June 2020; Accepted: 24 July 2020; Published: 29 July 2020

Featured Application: The proposed algorithm can be used to forecast short-term power fluctuations of a shipborne photovoltaic system, improve the power prediction accuracy of marine photovoltaic (PV) systems, provide a more reliable basis for the design of power prediction control methods and microgrid stability control, and can also be used to solve ship dispatching problems for power grids. In addition, PV systems that rely on MPPT (Maximum Power Point Tracking) control can also benefit from the proposed structure.

\begin{abstract}
When a solar ship is navigating in the ocean, the swaying motion of a photovoltaic panel will affect the output power of the photovoltaic (PV) power generation system more frequently and violently. In addition to considering multiple climatic factors, this paper also adopts a ship swaying motion and radiation level of sunlight to establish a suitable calculation model for the output power of photovoltaic systems, which are rarely considered at the same time in previous studies, and also to make ultrashort-term power predictions. Furthermore, this paper proposes a multilayer heterogeneous particle swarm optimization (PSO) algorithm to design the weights and thresholds of a long short-term memory (LSTM) neural network to improve the accuracy of forecasting the changes of a photovoltaic panel's angle, which is used for accurate power output prediction for the purpose of power planning. The case analysis shows the effectiveness of the algorithm, which provides a more reliable method for designing a power prediction system.
\end{abstract}

Keywords: shipborne solar system; long short-term memory; multilayer-heterogeneous PSO photovoltaic power calculation model; power fluctuation forecasting

\section{Introduction}

With the gradually increasing penetration rate of new energy sources, the development of marine renewable energy has become a hot research topic at both home and abroad, where green solar power, as one of the solutions, will be of great significance for energy saving and emission reduction in the field of environmental transportation. In a land micro-grid, solar power generation is only affected by natural conditions, but shipborne solar systems, such as a mobile micro-grid, are not only affected by natural conditions, but also by the navigation and sway of the ship itself. Such a system's power generation characteristics are more complex and changeable, even when the ship is parked. Due to the sway of the hull, the output of photovoltaic power generation system is constantly changing. Similarly, rapid and random photovoltaic (PV) panel fluctuations add a non-ignorable difficulty to PV power forecasting and calculation [1,2]. The power system in a ship featuring solar photovoltaic power generation is a complex system involving multiple variables and mutual coupling between 
various variables. It is necessary to fully consider the impact of ship motion and ship sway on solar output and accurately estimate the change of photovoltaic power, which has become a key problem for solar energy used on ships.

According to the existing research, when calculating the shipborne photovoltaic output power, the current methods ignore the significant impact of ship movement on PV systems, typically calculating the output power of the photovoltaic system by assuming a fixed photovoltaic tilt $[3,4]$. In the traditional mathematical model of a shipborne PV system, the inclination angle is not seen as a variable to calculate the output power. Research on the effect of a varying tilt angle toward photovoltaic output was carried out in [5-8], where the change of PV inclination affected the control method based on MPPT (Maximum Power Point Tracking) and the power planning, scheduling, and management of the ship's grid. This variable is also a significant parameter to forecast short-term power fluctuations in order to achieve an accurate reference for proactive frequency control [3]. Additionally, power prediction methods based on tilt angle estimation are also relatively rare, and it is difficult to accurately predict the photovoltaic power when affected by multiple uncertainties, which is not conducive to calculating a series of stability control problems.

Recently, machine learning has become an effective technology for solving nonlinear complex problems in systems. Golestaneh et al. focused on a short-term nonparametric probabilistic theory of solar power prediction which uses an extreme learning machine (ELM) [9]. A long short-term memory (LSTM) structure related to the forecasting problem for power was proposed in [10]. Wang and Sol et al. proposed an input delay neural network to analyze and forecast the feature of photovoltaic panel motion posture [11-13]. This approach had higher prediction accuracy and smaller prediction error. It is noticeable that the generalization ability of this structure is not strong enough, which means that the prediction effect is still unable to meet the actual engineering requirements. Additionally, refs. [14-16] features a study on using wavelet transform and support vector machine (SVM) algorithms to predict wind power. The above method is usually applied in the scenario of long-term power dispatching, where the methods are tested in a time span from $30 \mathrm{~min}$ to a few hours. However, for control operations with response levels in the second level, short-term accurate power prediction is necessary for a control strategy to achieve a better effect. The researchers in [3] used an ensemble-based ELM forecaster to predict power output, but the prediction time was too short and the response speed of the motor was relatively high, if the energy storage system is to work with the coordinated control of a ship's motor, the method mentioned in this article is insufficient.

Aiming to improve the accuracy of power fluctuation forecasting by means of predicting the photovoltaic panel tilt angle, this paper proposes an improved multi-layer heterogeneous particle swarm optimization (MHPSO) algorithm to optimize the parameters of long short-term memory (LSTM) neural networks. The concept of attracting particles is applied in a multi-layer heterogeneous PSO structure, and the speed update equation is updated to make the optimization performance of the particle swarm optimization algorithm more complete, which also improves the optimization effect on the selection of initial parameters of the LSTM structure, combining to build an improved MHPSO-LSTM model to forecast the output power of a PV system. The prediction time can reach $10 \mathrm{~s}$, which can reduce the requirements for motor and energy storage response time in the algorithm's applications for power generation control.

In this work, we use measured data from level three and five sea states as input to predict the movement results of the photovoltaic panel. The solar illuminance data are sourced from a ship route from the north of China to the east of Arabia. The radiation amount, corrected by the latitude and longitude, is used for calculation in the photovoltaic system output power prediction model. The aim of this paper is to forecast rapid power fluctuations caused by the swing of shipborne photovoltaic panels traveling on the sea and the uncertainty of renewable energy in a short time (within $10 \mathrm{~s}$ ).

The contributions of this paper are as follows: This article provides a more reliable method for calculating the output power of photovoltaic systems on ships following known routes. At the same time, by predicting the inclination of the PV panel, the output power is forecasted in the short-term. 
(1) Aiming at shipborne PV systems, this paper proposes a power calculation model for shipborne photovoltaic panels. Sea wave fluctuation has a non-negligible effect on the output power of the PV system. The measured roll and pitch are reduced to one single angle through a rotation matrix, which is used as an important variable in our model. Then, we calculate the output power through the proposed model and the proposed model is tested with a solar ship swing platform.

(2) The accuracy of short-term power prediction is of great significance to power control, so we propose to predict and calculate the power of a photovoltaic system by predicting the inclination of the photovoltaic panel. We use an improved LSTM algorithm to complete this work.

(3) According to the year-round weather data of known routes, the solar radiation along the route should be corrected for. The radiation is also variable, according to the season, latitude, and longitude, which provides a reliable reference source for the power scheduling of shipborne PV system.

This paper is organized as follows: Section 2 establishes the inclination motion model of the shipborne photovoltaic panel and Section 3 presents the LSTM neural network. Section 4 presents the improved MHPSO algorithm and Section 5 describes the MHPSO structure for optimizing the weights of the LSTM neural network and discusses the proposed structure of the MHPSO-LSTM algorithm and its effectiveness in the prediction of the pitch angle of the photovoltaic panel. In addition, we build a more accurate mathematical model of photovoltaic power based on the proposed prediction algorithm and make ultrashort-term power predictions. Finally, some conclusions are drawn in Section 6.

\section{Model of Ship Photovoltaic Panel Swing Motion}

Here, the solar photovoltaic modules are all horizontally arranged on the ship deck, and the photovoltaic modules on the ship will move together with the ship's swing. While sailing in the ocean, due to the effects of wind, waves, and ocean currents, this will produce six degrees of freedom (DOF) movements, namely, surge, sway, heave, roll, pitch, and yaw [17]. This section introduces the calculation model of the spatial motion variation of shipborne photovoltaic panels under the influence of rolling and pitching when the ship is sailing.

The three-dimensional coordinate transformation model in space takes the common transformation between the world and the camera coordinate system as an example. In the rotation matrix of the hull, the Euler angle is rotated by rotating the rigid body around the axis of the origin [18]. As shown in Figure 1, blue coordinates is the starting system $(x, y, z)$, the red is the coordinate system after rotation $\left(x^{\prime}, y^{\prime}, z^{\prime}\right)$. Taking a vector $\overrightarrow{O P}$ in a three-dim coordinate system to rotate around the $z$ axis by the angle of $\theta$ as an example to derive the process of converting the Euler angle of rotation from the world coordinate system to the camera coordinate system, as shown in Figure 2.

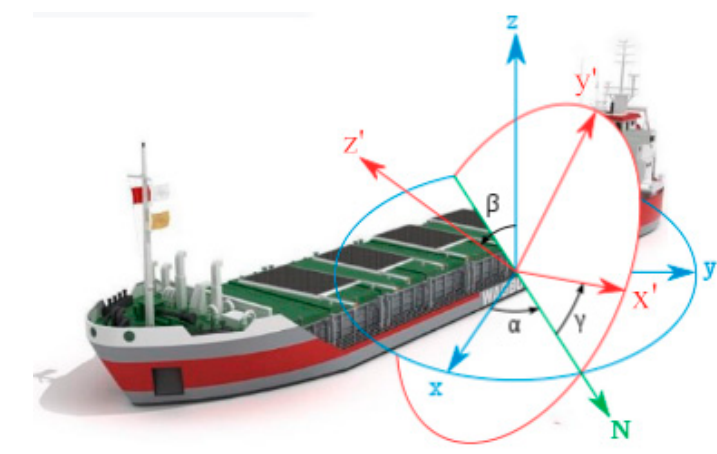

Figure 1. Rotation matrix of a three-dimensional space coordinate system. 


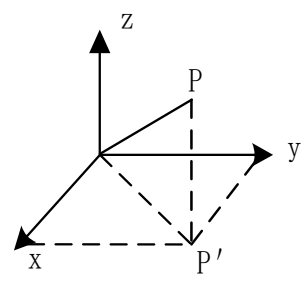

(a) Vectors in three dimensions.

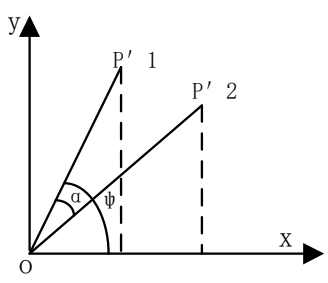

(b) xOy plane.

Figure 2. Rotation process of the Euler angle.

In Figure $2 \mathrm{a}, O P$ forms $P^{\prime}$ as a projection on the $\mathrm{xOy}$ plane, rotating around the $\mathrm{Z}$ axis, equivalent to $O P^{\prime}$ s projection on the $\mathrm{xOy}$ plane, which rotates around the origin. As shown in Figure $2 \mathrm{~b}, O P^{\prime} 1$ rotates $\alpha$ degrees to $O P^{\prime} 2$ and $O P^{\prime} 1=O P^{\prime} 2$ [13].

$$
\left\{\begin{array} { l } 
{ P ^ { \prime } 1 = ( x 1 , y 1 ) } \\
{ P ^ { \prime } 2 = ( x 2 , y 2 ) }
\end{array} \quad \left\{\begin{array}{l}
x 1=O P^{\prime} 1 \cos \psi \\
y 1=O P^{\prime} 1 \sin \psi
\end{array}\right.\right.
$$

It can be obtained that the rotation matrix of $\beta$ angle rotation around the $Z$ axis is

$$
R_{Z}(\beta)=\left[\begin{array}{ccc}
\cos \beta & -\sin \beta & 0 \\
\sin \beta & \cos \beta & 0 \\
0 & 0 & 1
\end{array}\right]
$$

The rotation from the world coordinate changed to the camera coordinate shows that the rotation angle around the $\mathrm{Z}$ axis is yaw, where then the rotation angle twirls around the $\mathrm{Y}$ axis of the coordinate system after rotation, and then the roll angle is rotated around the $\mathrm{X}$ axis of the coordinate system after rotation, where $\beta=$ roll, $\gamma=$ pitch, $\alpha=$ heave, therefore the final rotation angle matrix is as given follows:

$$
\begin{gathered}
R_{z} R_{y} R_{x}=\left[\begin{array}{ccc}
\cos (\alpha) & -\sin (\alpha) & 0 \\
\sin (\alpha) & \cos (\alpha) & 0 \\
0 & 0 & 1
\end{array}\right]\left[\begin{array}{ccc}
\cos (\gamma) & 0 & \sin (\gamma) \\
0 & 1 & 0 \\
-\sin (\gamma) & 0 & \cos (\gamma)
\end{array}\right]\left[\begin{array}{ccc}
1 & 0 & 0 \\
0 & \cos (\beta) & -\sin (\beta) \\
0 & \sin (\beta) & \cos (\beta)
\end{array}\right] \\
=\left[\begin{array}{ccc}
\cos (\alpha) \cos (\gamma) & \cos (\alpha) \sin (\gamma) \sin (\beta)-\sin (\alpha) \cos (\beta) & \cos (\alpha) \sin (\gamma) \cos (\beta)+\sin (\alpha) \sin (\beta) \\
\sin (\alpha) \cos (\gamma) & \sin (\alpha) \sin (\gamma) \sin (\beta)+\cos (\alpha) \cos (\beta) & \sin (\alpha) \sin (\gamma) \cos (\beta)-\cos (\alpha) \sin (\beta) \\
-\sin (\gamma) & \cos (\gamma) \sin (\beta) & \cos (\gamma) \cos (\beta)
\end{array}\right]
\end{gathered}
$$

Next, one needs to calculate the angle $\delta$ change between the photovoltaic panel and the sea level after generating roll angle $R_{X}$ and pitch angle $R_{Y}$. According to the Equations (3) and (4), we can get $\delta$ :

$$
\begin{aligned}
& \delta=\mathrm{a} \tan 2(r 32, r 33) \\
& =\arctan \left(\frac{\cos (\gamma) \sin (\beta)}{\cos (\gamma) \cos (\beta)}\right) \quad-\pi<\delta<\pi
\end{aligned}
$$

The numerical values of the roll and pitch will be forecasted by the proposed algorithm in the next section.

\section{LSTM Neural Network}

In this section, we discuss an improved neural network-based method for predicting the inclination of photovoltaic panels for the short-term power prediction of PV systems, recurrent neural network (RNNs) are sequence-based models which are able to form the temporal correlations between previous experiences and the current state [19]. Nevertheless, it is difficult to learn long-term dependence due to the disappearance or eruption of gradients. In [19], the authors firstly proposed a long short-term 
memory (LSTM) algorithm, where a store cell was draw in by Gers et al. LSTM has three carefully designed structures called "gates", named the input, forget, and output gates, respectively, which have ability to remove or add information to the state of the cell. Gates are a way to select which information is used. With an additional forget gate, this model avoids the problem of gradient disappearance while learning to depend on information for a long range; thereby, adding a structure which is a memory unit to the hidden layer of the time-recurrent neural network to remember past information.

The LSTM model can be described by Equations (5)-(10):

$$
\begin{gathered}
i_{t}=\operatorname{sigmoid}\left(W_{h i} h_{t-1}+W_{x i} x_{t}\right) \\
f_{t}=\operatorname{sigmoid}\left(w_{h f} h_{t-1}+W_{h f} h_{t-1}\right) \\
c_{t}=f_{t \odot} c_{t-1}+i_{c \odot} \tanh \left(W_{X C} h_{t-1}+W_{h c} h_{t-1}\right) \\
o_{t}=\operatorname{sigmoid}\left(W_{X C} x_{t}+W_{h c} h_{t-1}\right) \\
h_{t}=o_{t \odot} \tanh \left(c_{t}\right) \\
W_{h i}, W_{x i}, W_{h f}, W_{X C} \in R^{1000 \times 2000}
\end{gathered}
$$

The weight matrices $W_{h i}, W_{x i}, W_{h f}, W_{X C}$, and $W_{h c}$ act on behalf of the LSTM structure activation functions, which will affect loss function, and $i_{t}, f_{t}$, and $o_{t}$ are the input, forget, and output gates of the LSTM model, respectively. The first step in the LSTM is to decide what message is discarded from the cell state. This step is made through a layer called the forget gate, and then the output gate is used to control how much of the cell state $c_{t}$ will be output to the current output value $h_{t}$ of the LSTM model. Here, $\odot$ stands for an element-wise multiplication, $h_{t}$ is the output gate, $h_{t-1}$ is the output in the previous moment from the LSTM, and the elements that should be updated are determined by it. The common initialization for $h_{t}$ is zero, sigmoid and tanh are the activation functions. Here, $c_{t}$ is the cell unit on behalf of the current state. The initialization of the model parameters of the LSTM model are stochastic, which leads to a reduction of the nonlinear learning of the existing parameter optimization methods.

Figure 3 shows that the repeating module in the LSTM neural network contains four interactive layers. All RNNs have a chained form of repeating neural network modules [20]. In a standard RNN, this repeated module only has a very simple structure, such as a tanh layer. Every arrow line delivers an entire vector from the output to the input of one of the other nodes, respectively. The red circles at the end denote the pointwise operations, such as vector multiplication, and the matrix in yellow is the learned neural network layer. The combined lines indicate the connection of vectors, and the separate lines indicate that the content is copied and then distributed to another locations.

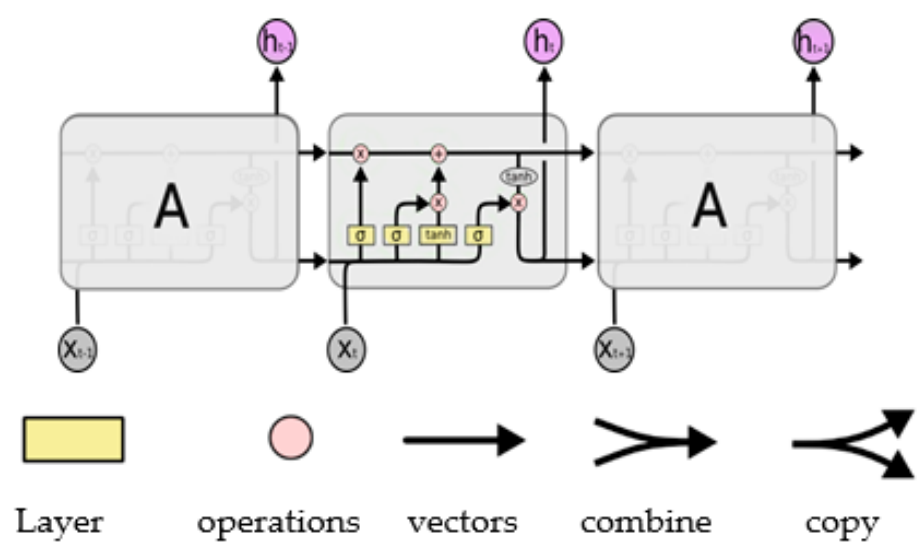

Figure 3. Repeating configuration in the long short-term memory (LSTM) neural network interactive layer. 


\section{MHPSO-LSTM}

As shown in Equations (11) and (12), PSO, as an intelligent optimization structure, can be used to simulate the particles in each bird's search space, during which the speed is adjusted according to the two equations until the conditions converge. Affected by its own best past position $\hat{y}^{t}$ and the best past position $y_{i, j}^{t}$ of the entire group or neighbors, particles use their own experience and the best experience of their companions to decide their next movement.

$$
\begin{gathered}
V_{i, j}^{t+1}=\omega V_{i, j}^{t}+c_{1} r_{1, i, j}^{t}\left(\hat{y}_{i}^{t}-x_{i, j}^{t}\right)+c_{2} r_{2, i, j}^{t}\left(y_{i, j}^{t}-x_{i, j}^{t}\right) \\
x_{i, j}^{t+1}=x_{i, j}^{t}+V_{i, j}^{t+1}
\end{gathered}
$$

$V_{i, j}^{t+1}$ means the velocity of some particles at a specific time, namely $i$ in $j$ at $t$, and $x_{i, j}^{t}$ is the position of $i$ at $t$. Additionally, $\omega$ is an inertia weight, representing the memorization of particles at a changed position, and the global optimization performance and local optimization performance can be adjusted by adjusting the size of $\omega$. Additionally, $c 1$ and $c 2$ are constant factors related to acceleration, meanwhile $\hat{y}^{t}$ is an individual optimal scheme for one particle at a time, $y_{i, j}^{t}$ is a global optimal solution known at $t$, and $r_{1,2, i, j}^{t}$ are random numbers.

This paper proposes a multi-layer heterogeneous topology dynamic particle swarm optimization algorithm. In this sense, an algorithm named MHPSO has been developed, with the core goal of establishing vertical interaction between various layers. The particle swarm structure is shown in Figure 4.

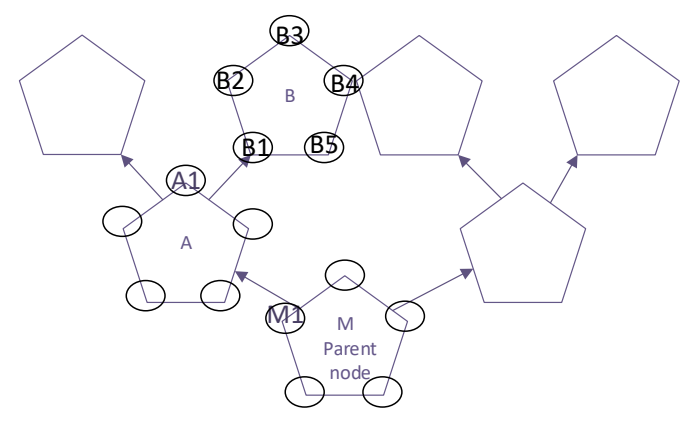

Figure 4. Improved particle swarm algorithm structure.

The number of particles in each layer are the same, and the algorithm randomly assigns every five particles in the population to a loop, in which a rule tree has taken shape. The total fitness values formed by all the particles in the loop finally decide the quality of each and every loop, where the advantages and disadvantages of the loop determine the position of the loop in the tree and better loops are in a higher position in the tree. During the operation of the algorithm, the position of the loop is dynamically adjusted according to the advantages and disadvantages of the loop. The particles in the parent node within this structure are used as the attracting ones and the particles in the offspring nodes are also their attracting particles. The attracting particles of the top ones are all on the same layer and show the characteristics of better adaptability to the population. In addition to moving to the optimal position in the local and global spaces, they also move to the place where the attractor is located. As shown in Figure 4, the attractor of particle A1 includes 5 particles (B1-B5). The attractor of particles (such as M1) in the loop at the parent node is just 4 other particles in one loop.

In the improved algorithm, the update velocity Equation (13) contains additional terms for attracting particles:

$$
\begin{aligned}
& V_{i, j}^{t+1}=\omega V_{i, j}^{t}+c_{1} r_{1, i, j}^{t}\left(\hat{y}^{t}-x_{a, j}^{t}\right)+c_{2} r_{2, i, j}^{t}\left(y_{i, j}^{t}-x_{i . j}^{t}\right)+ \\
& \sum_{a=1}^{A_{j}} c_{3} r(i)_{a, j}^{t}\left(x(i)_{a, j}^{t}-x_{i, j}^{t}\right) / A_{j}^{i}
\end{aligned}
$$


In order to improve the robustness of the algorithm, the particle attraction coefficient $r(i)_{a, j}^{t}$ uses new calculation methods to ensure that the influence of the attractor on each particle is balanced.

(1) When $S(i)_{a, j}^{t} \leqq \bar{S}(i)_{j}^{t}$,

$$
r(i)_{a, j}^{t}=r_{\min }^{t}+\frac{\left(r_{\max }^{t}-r_{\min }^{t}\right)\left(S(i)_{a, j}-S(i)_{\min , j}\right)}{S(i)_{j}^{t}-\bar{S}(i)_{\min , j}^{t}}
$$

(2) When $S(i)_{a, j}^{t} 1>\bar{S}(i)_{j}^{t}$

$$
r(i)_{a, j}^{t}=r_{\min }^{t}+\frac{\left(r_{M A X}^{t}-r_{M I N}^{t}\right)\left(S(i)_{M A X, j}^{t}-S(i)_{a, j}^{t}\right)}{S(i)_{M A X, j}^{t}-\bar{S}(i)_{j}^{t}}
$$

where $r_{\min }^{t}$ and $r_{\max }^{t}$ are the minimum and maximum attractor coefficients, $S(i)_{\min , j}^{t}$ and $S(i)_{\max , j}^{t}$ show particle $i$ with both the minimum and maximum values, and $S \overline{(i)}_{j}^{t}$ is the average coordinate position of all attracted particles.

The main purpose of the algorithm is to use the improved multi-layer heterogeneous particle swarm optimization algorithm to optimize the weights of the LSTM structure, as shown in Figure 5. Table 1 provides the parameter set, where the ultimate goal is to assign the obtained optimal particles to the connection weights of the LSTM structure. The specific solution steps are as follows.

Step 1: Initialize the LSTM network structure with the main goal to determine the number of neurons. PSO includes the size, overall level, and learning factors, and the particle positions and velocities are random. According to the mean square error (MSE) of the data set used by particle $i$ to search for the local optimal solution and the global optimal solution during the training process, this is expressed as follows:

$$
\begin{gathered}
M S_{i}=\frac{1}{p} \sum_{y=1}^{P} \sum_{j=1}^{N}\left(d_{i j}-y_{i j}\right)^{2} \\
M S E_{g}=\min _{i=1}^{n}\left(M S E_{i}\right)=\min _{i=1}^{n} \frac{1}{p} \sum_{y=1}^{P} \sum_{j=1}^{N}\left(d_{i j}-y_{i j}\right)^{2}
\end{gathered}
$$

where $M S E_{g}$ is the fitness function, and $d_{i j}$ and $y_{i j}$ are the predicted and true values, respectively.

Step 2: Calculate the quality of the loops and establish the particle evaluation function. The fitness function in the population is defined as

$$
\text { Fit }_{i}=\frac{1}{n} \sum_{i=1}^{n}\left|\frac{Y_{i}-y_{i}}{y_{i}}\right|
$$

where $n$ stands for the size of population, $Y_{i}$ is the forecast value of the sample, and $y_{i}$ is the actual output.

Step 3: Calculate and sort each group of fitness values based on Equation (18) and upgrade the local and global optimal positions.

Step 4: If the end condition of the iteration (optimal position) is reached, move back to Step 3 and continue to iterate.

Step 5: Allocate the acquired optimal particles to the LSTM network-related weight. Train the LSTM network prediction model and output the time series prediction optimal solution. 


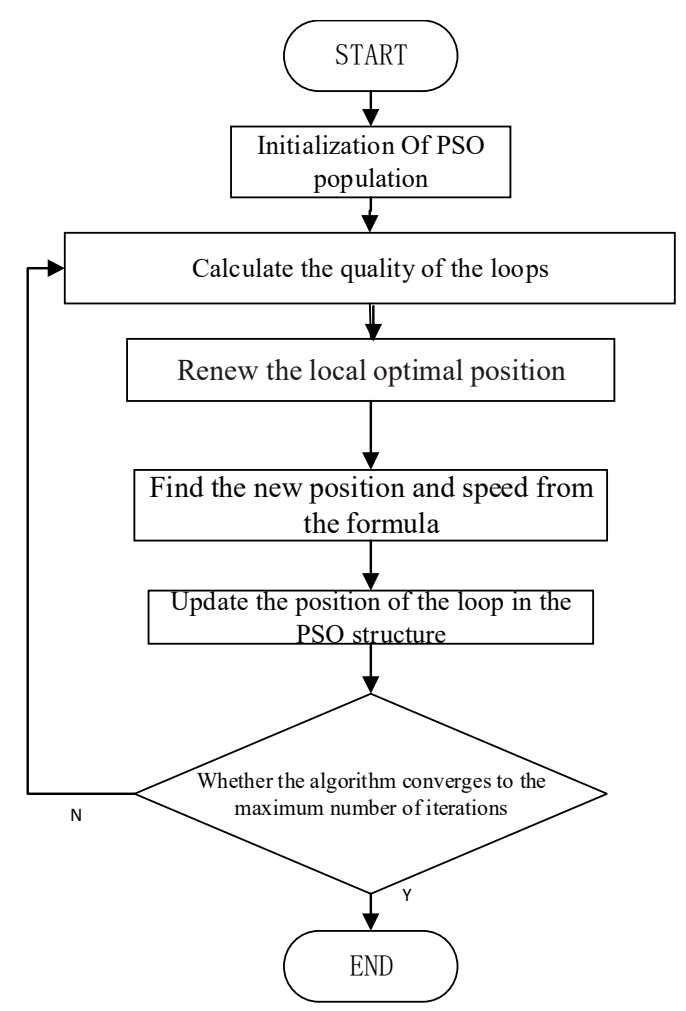

Figure 5. Multilayer heterogeneous particle swarm optimization (MHPSO) algorithm flow chart.

Table 1. Parameter summary.

\begin{tabular}{cccccccc}
\hline Parameter & $\omega$ & $c 1$ & $c 2$ & $r 1$ & $r 2$ & $r_{\max }^{t}$ & $r_{\min }^{t}$ \\
\hline PSO & 0.6 to 0.2 & 2.5 to 0.5 & 0.5 to 2.5 & $\begin{array}{c}\text { Standard normal } \\
\text { distribution }\end{array}$ & $\begin{array}{c}\text { Standard normal } \\
\text { distribution }\end{array}$ & - & - \\
\hline MHPSO-LSTM & 0.6 to 0.2 & 2.5 to 0.5 & 0.5 to 2.5 & $\begin{array}{c}\text { Standard normal } \\
\text { distribution }\end{array}$ & $\begin{array}{c}\text { Standard normal } \\
\text { distribution }\end{array}$ & 1.3 & 0.4 \\
\hline
\end{tabular}

\section{Prediction of Photovoltaic Output Power Based on MHPSO-LSTM}

\subsection{Photovoltaic Panel Motion Prediction}

In this section, we analyze and discuss the improved MHPSO-LSTM method and the effect toward forecasting the inclination of the photovoltaic panel on board to predict the change of PV power output within $10 \mathrm{~s}$. The proposed LSTM structure includes structural optimization and photovoltaic panel motion prediction. The flow chart of the algorithm structure is shown in Figure 6. 


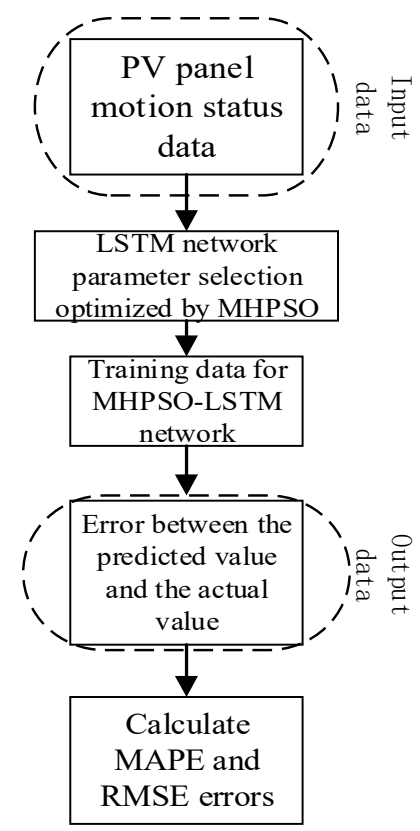

Figure 6. Neural network structure of the LSTM model based on MHPSO. MAPE: Mean absolute percentage error. RMSE: Root mean square error.

In this work, we forecast the future motion data of a PV panel swing state through known historical swing data. In order to verify the precision and advantage of the MHPSO-LSTM method, we used a BP (back propagation) neural network, Kalman filter, LSTM neural network, PSO-LSTM network, and MHPSO-LSTM network model for comparative experiments. We used Equation (19) to normalize the data to a $[0,1]$ interval in order to converge quickly. For the evaluative indicators of the forecast results, we took the mean absolute percentage error (MAPE) and root mean square error (RMSE), where $\hat{x}_{t}$ is the prediction results of PV panel motion and $x_{t}$ is the actual values of panel motion.

$$
\begin{gathered}
M_{i}=\frac{x_{i}-x_{\min }}{x_{\max }-x_{\min }} \\
M A P E=\frac{1}{n} \sum_{i=1}^{n}\left|\frac{\hat{x}_{t}-x_{t}}{x_{t}}\right| \times 100 \% \\
\text { RMSE }=\sqrt{\frac{\sum_{j=1}^{n}\left(\hat{x}_{t}-x_{t}\right)^{2}}{x_{t}}}
\end{gathered}
$$

In the simulation process, the measured data input was sourced from level 5 and level 3 sea states. The sea state is divided into 10 levels according to the sea surface fluctuations, and the shape of the wave peaks and the degree of breakage, which is expressed as 0-9 level from low to high. The sampling frequency of the measured PV tilt change was $0.193 \mathrm{~s}$, the quantity of training samples was 250 , and the number of predicted samples was 50, respectively. Additionally, the forecast duration was $9.65 \mathrm{~s}$, and in the improved LSTM structure, the hidden layers were set to 3, the number of neurons was 50, number of iterations was 200, epoch times was 200 and the output data were the tilt angles of the photovoltaic panel, include the roll and pitch, which was then calculated by the matrix proposed in the second section for the Z-axis rotation matrix to get $\delta$.

The above models (Kalman, BP, PSO-LSTM, LSTM, and MHPSO-LSTM) were used to predict the inclination of the PV panel under the level 5 and level 3 sea states. Figure 7 shows a comparison of the angle prediction results of the different prediction methods under the fifth-level sea state. Figure 8 shows a comparative group under a level 3 sea state. 


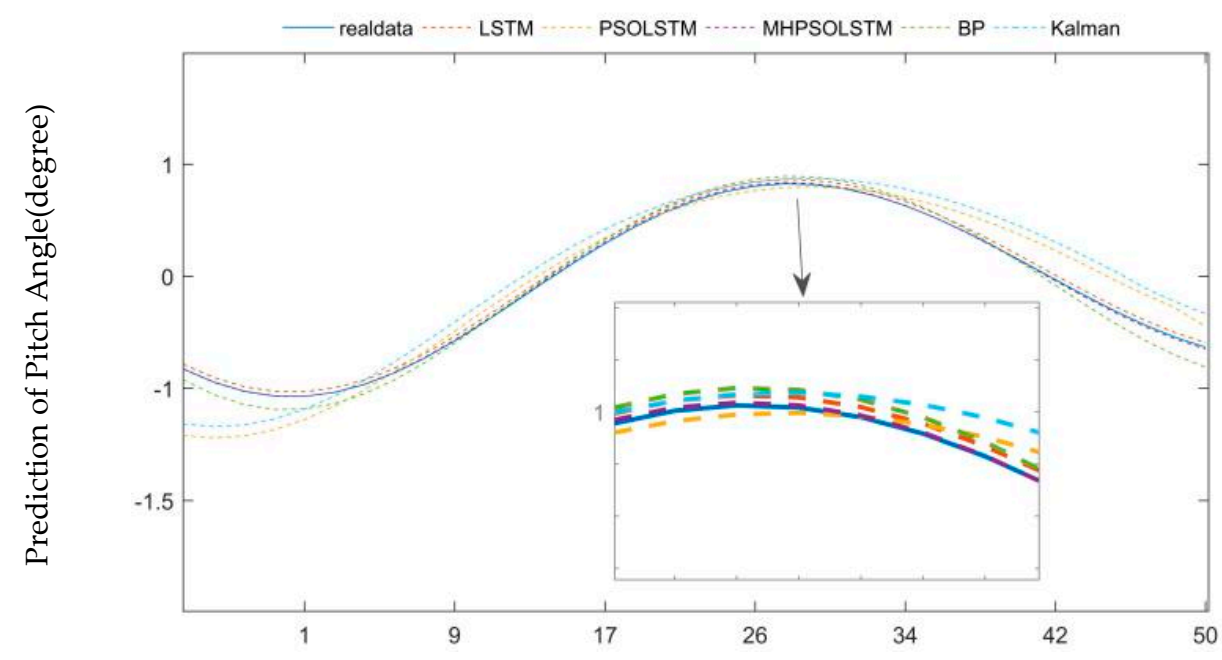

(a) Index of data

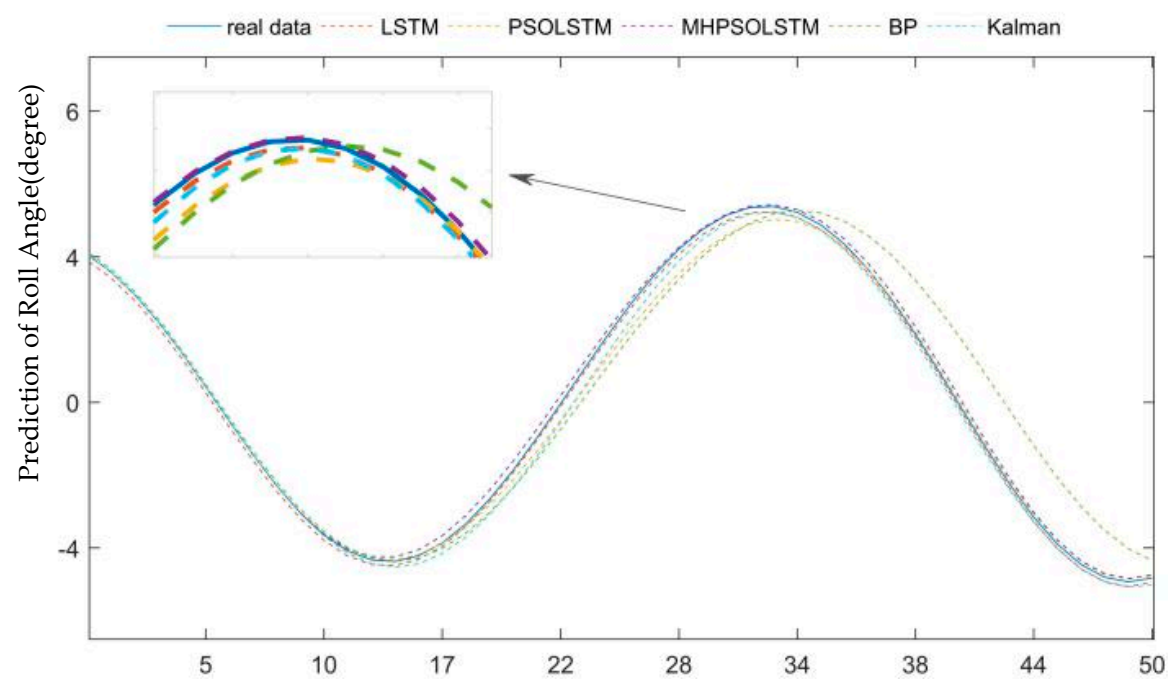

(b) Index of data

Figure 7. Prediction results of the five models under a level 5 sea state.

Figure $7 \mathrm{a}$ indicates the prediction curve of the pitch angle under a level 5 sea state, which corresponds to "pitch" in Equation (4). Similarly, Figure 7b corresponds to "roll" in Equation (4).

Figure 9 shows that the prediction error of the proposed PSO algorithm can be controlled to within \pm 0.1 degree. It can be seen in Table 2 that the values of the MAPE and RMSE are used for the forecast. From the comparison of the forecast results of the proposed methods, it can be seen that the MHPSO structure improves the global search ability of the PSO algorithm by strengthening the message sharing between different particles. 


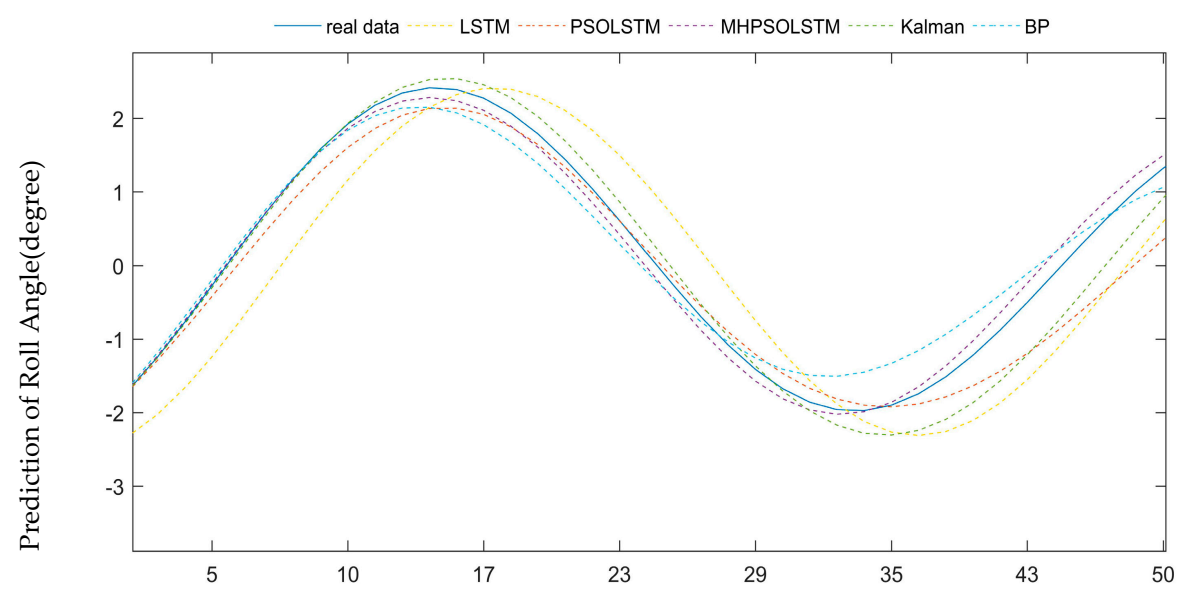

(a) Index of data.

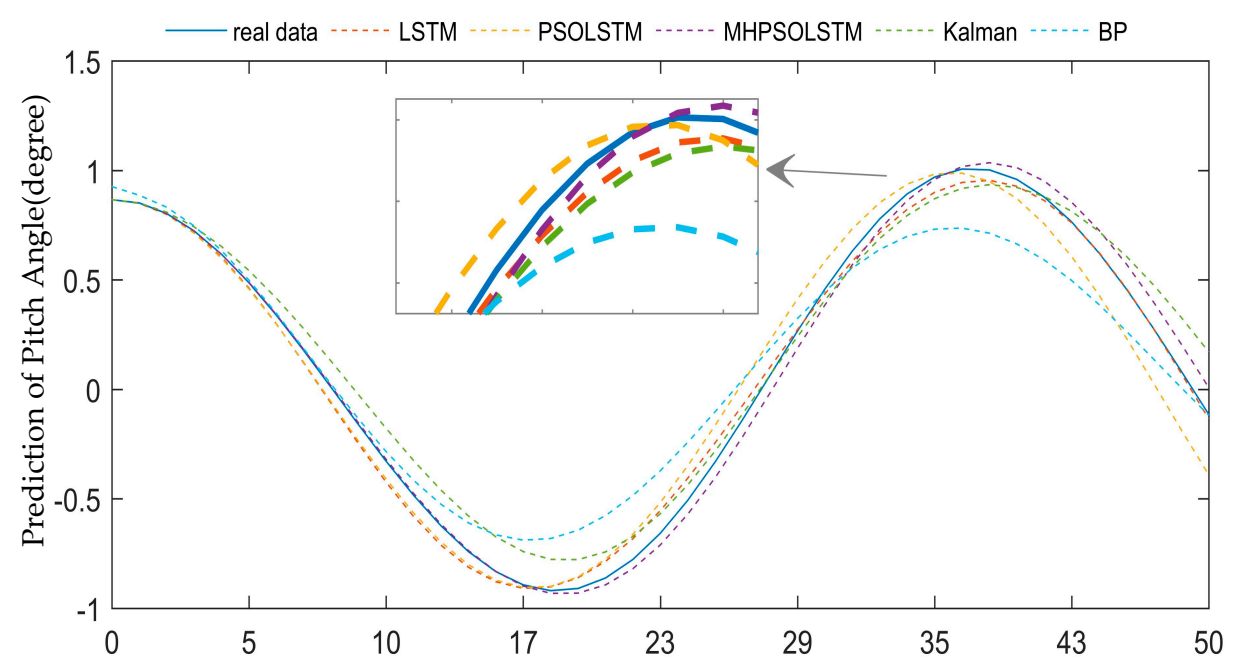

(b) Index of data

Figure 8. Prediction results of the five models under a level 3 sea state.

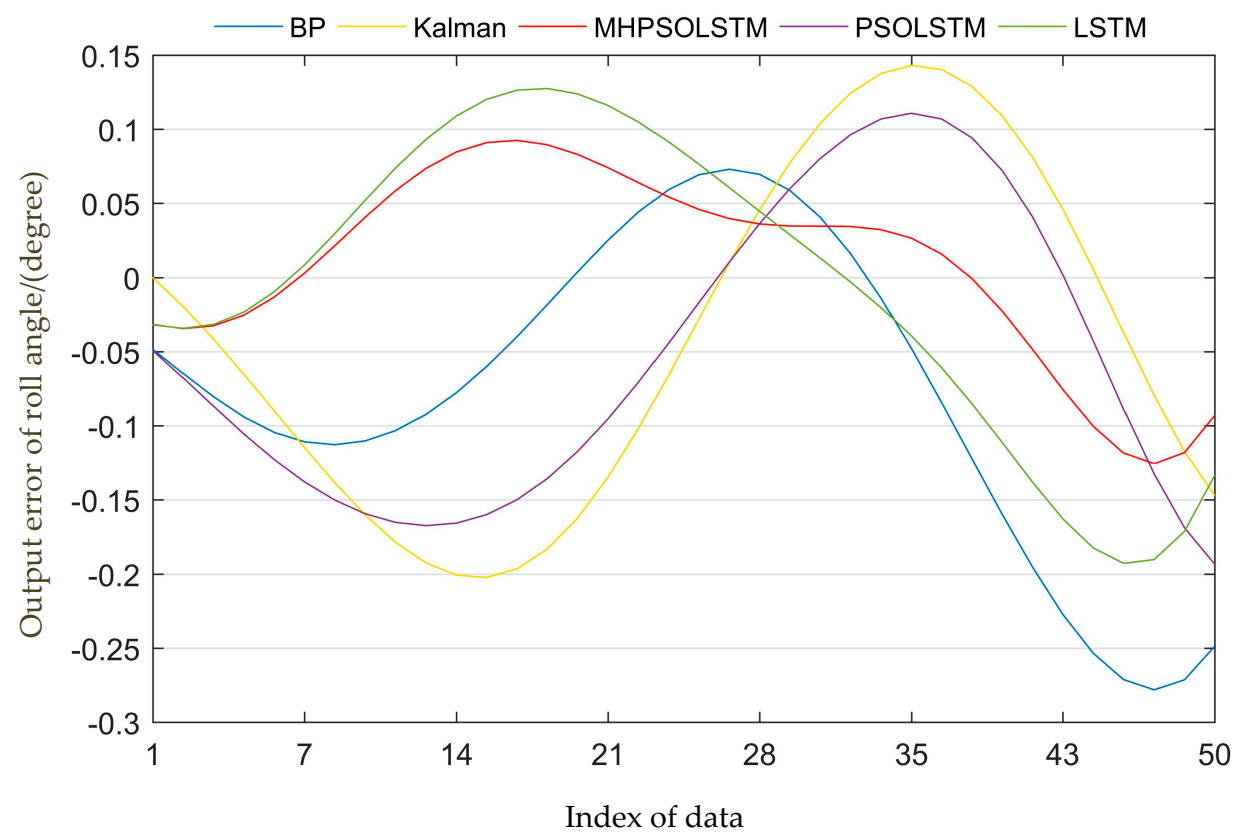

Figure 9. Comparison of the five methods for the photovoltaic (PV) panel angle prediction error. 
Table 2. The MAPE and RMSE values of the 5 methods for forecasting the PV panel.

\begin{tabular}{|c|c|c|c|c|c|c|}
\hline \multicolumn{2}{|c|}{ Method } & BP NN & Kalman & LSTM & PSO-LSTM & MHPSO-LSTM \\
\hline Roll & МAPE\% & 15.54 & 15.74 & 13.65 & 7.36 & 5.11 \\
\hline Angle (level 5) & RMSE & 0.0328 & 0.0384 & 0.0385 & 0.0366 & 0.0116 \\
\hline Pitch & МAPE\% & 12.51 & 13.68 & 12.52 & 8.87 & 2.98 \\
\hline Angel (level 5) & RMSE & 0.0425 & 0.0476 & 0.0487 & 0.0312 & 0.0312 \\
\hline Roll & MAPE\% & 16.58 & 18.65 & 18.63 & 11.84 & 6.43 \\
\hline Angel (level 3) & RMSE & 0.1911 & 0.1974 & 0.1832 & 0.1611 & 0.0966 \\
\hline Pitch & MAPE\% & 16.67 & 16.45 & 15.76 & 10.56 & 5.61 \\
\hline Angel (level 3) & RMSE & 0.1798 & 0.1723 & 0.1678 & 0.1421 & 0.0834 \\
\hline
\end{tabular}

Table 3 demonstrates the convergence time (s) of five methods under difference sea state, it costs $0.1387 \mathrm{~s}$ to convergence for the improved LSTM structure, which is $4 / 5$ to $1 / 3$ of other algorithms in CPU times under AMD Ryzen5-3600X condition, combined with the improvement in accuracy which is stated in Table 2, the performance of the proposed algorithm has been significantly improved.

Table 3. The convergence time (s) of the 5 methods for forecasting the PV panel.

\begin{tabular}{cccccc}
\hline Sea State/Method & BP NN & Kalman & LSTM & PSO-LSTM & MHPSO-LSTM \\
\hline Level 5 & 0.3010 & 0.5127 & 0.1811 & 0.1542 & 0.1387 \\
Level 3 & 0.3010 & 0.5125 & 0.1810 & 0.1542 & 0.1387 \\
\hline
\end{tabular}

Since there are few studies on the application of a LSTM-based neural network for ship motion attitude forecasting, in the next step we will continue to apply the MHPSO-LSTM model to shipborne photovoltaic panel swing prediction.

\subsection{Multivariable Coupling Solar Photovoltaic Power Generation Model}

According to the estimation of available space being 300,000 tons for large oil tankers, it is expected that $2000 \mathrm{~m}^{2}$ of YGE145 series photovoltaic modules can be laid. Solar photovoltaic power generation systems include solar cell components, hybrid energy storage devices, controllers, and inverters. In this paper, a series of experiments were carried out on a solar ship swing platform. The device consisted of nine xenon lamps, eight solar photovoltaic panels (power factor: 0.861819573, module efficiency: 0.1451) and a six DOF swing table, as shown in Figure 10, which can simulate the movement of the ship at sea, such as the ship's heave, roll, and pitch, and determine the sunlight spreading coefficient and reflectivity in the shipboard photovoltaic power generation system. The artificial light intensity here was controlled to expanded to a $2000 \mathrm{~m}^{2}$ area as a scale model.

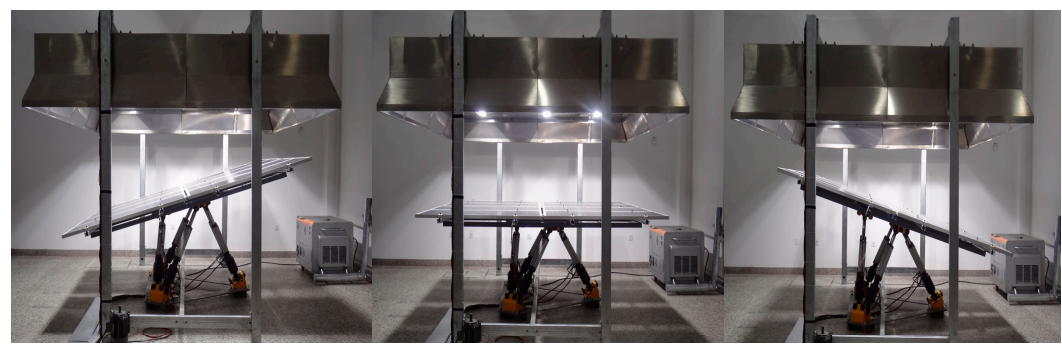

Figure 10. Solar ship swing platform.

The traditional marine photovoltaic power calculation model often only considers the changes in illumination, latitude, and longitude and does not take the PV panel's motion status as a non-negligible variable [21-23]. Under the traditional model, for ships with a fixed latitude and longitude, such as marine drilling platforms, the power change of a photovoltaic panel only depends on solar radiation 
intensity $I_{T}[24,25]$. In this paper, the change of the photovoltaic panel inclination angle was introduced into the ship's photovoltaic power calculation model and the output power of the photovoltaic system was predicted for $10 \mathrm{~s}$ via the improved MHPSO-LSTM algorithm. The improvement of the calculation model provides a reliable basis for the power planning and dispatching of a ship's power grid and is a control method based on prediction. The direct solar radiation on the swinging photovoltaic surface can be represented by Figure 11 .

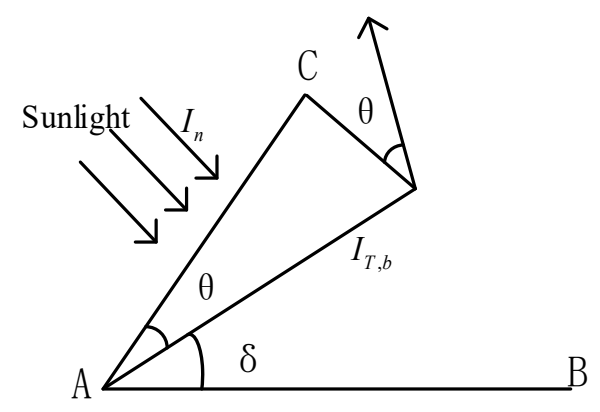

Figure 11. Radiation of inclined PV panels.

Since the amount of solar energy received on side $A C$ is equal to the amount of solar energy received on side $A B$, this can be denoted by Equation (22). The amount of tilted photovoltaic radiation can be expressed by Equations (23)-(26):

$$
\begin{gathered}
I_{T, b}=I_{n} \cos \theta \\
I_{T}=I_{T, b}+I_{T, d}+I_{T, r} \\
=I_{n} \cos \theta+I_{d}\left(\frac{1+\cos \delta}{2}\right)+\rho\left(\frac{1-\cos \delta}{2}\right) \\
I_{T, d}=(1+\cos \delta) / 2 \\
I_{T, b}=I_{n} \cos \theta \\
I_{T, r}=\rho(1-\cos \delta) I / 2
\end{gathered}
$$

where $\theta$ is the sun angle, $I_{T, d}$ is the scattered radiation, $I_{T, b}$ is the direct solar radiation, $I_{T, r}$ is the deck reflected radiation, $I_{n}$ is the value of the direct solar radiation incident on the surface, $I_{d}$ is the amount of scattered solar radiation on the horizontal plane, and $\rho$ is the ground reflectivity. Here, $\delta$ is the predicted value of the inclination of the deck, which is calculated in Section 5.

Since the solar illuminance directly affects the calculation result of the photovoltaic output, this paper corrects the coefficient $(\cos \theta)$ of the shipborne solar photovoltaic system based on the solar illuminance data from the north of China to the east of Arabia, provided by the GeoModel Solar Company, as shown in Figure 12.

$$
\begin{gathered}
\cos \theta=\cos \chi=\sin \varsigma \sin \lambda+\cos \varsigma \cos \lambda \cos \tau \\
\varsigma=23.4 \sin \left[360\left(\frac{d-80}{365.25}\right)\right]
\end{gathered}
$$

Where $\chi$ is the zenith angle, $\varsigma$ is the sun dip angel, $\lambda$ is the latitude of the ship, $\tau$ is the sun angel, and $d$ is the Julian days.

We select the latitudes zone between $1^{\circ}$ to $30^{\circ}$ as an example to calculate the daily average solar radiation of the target ship with different tilt angel $\delta$ as shown in Figure 13. It can be seen that under different latitudes and tilt angel, the amount of solar radiation is different. After the correction, the light intensity received by the photovoltaic panel on the deck of the tanker was considered, shown in Figure 14. The yellow, green, orange, and blue lines represent the solar radiation for the four seasons. 


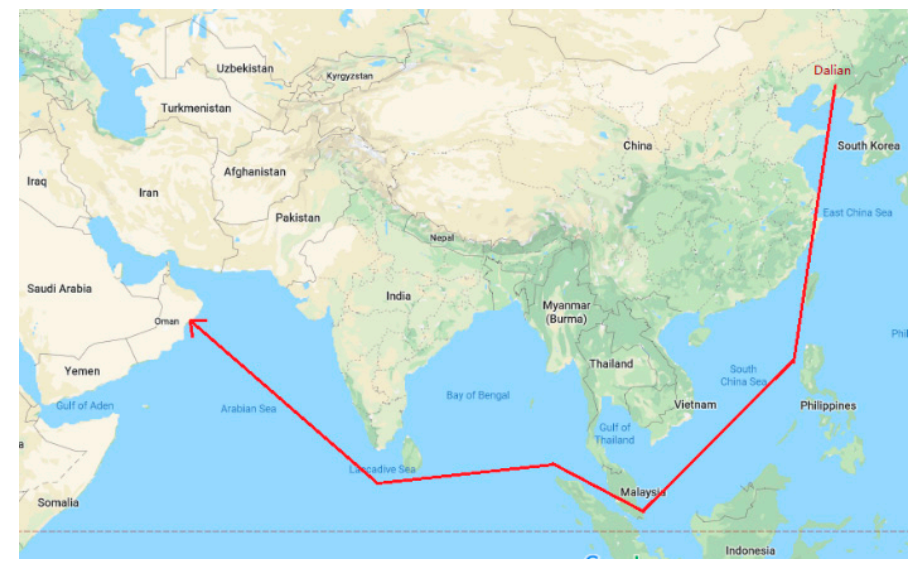

Figure 12. Route map.

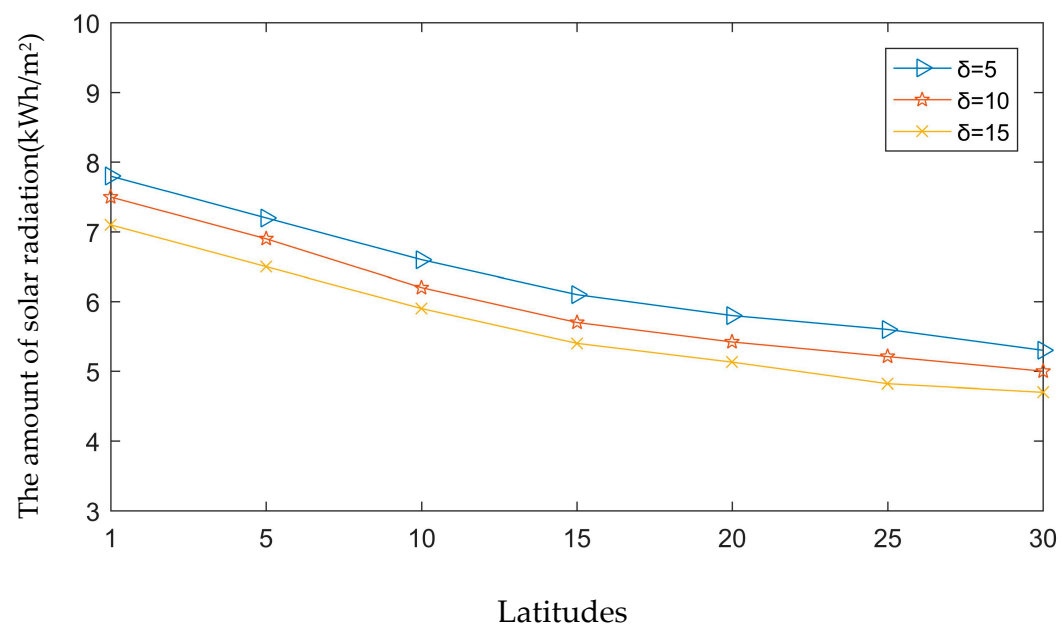

Figure 13. Total power generated by the PV system at various latitudes.

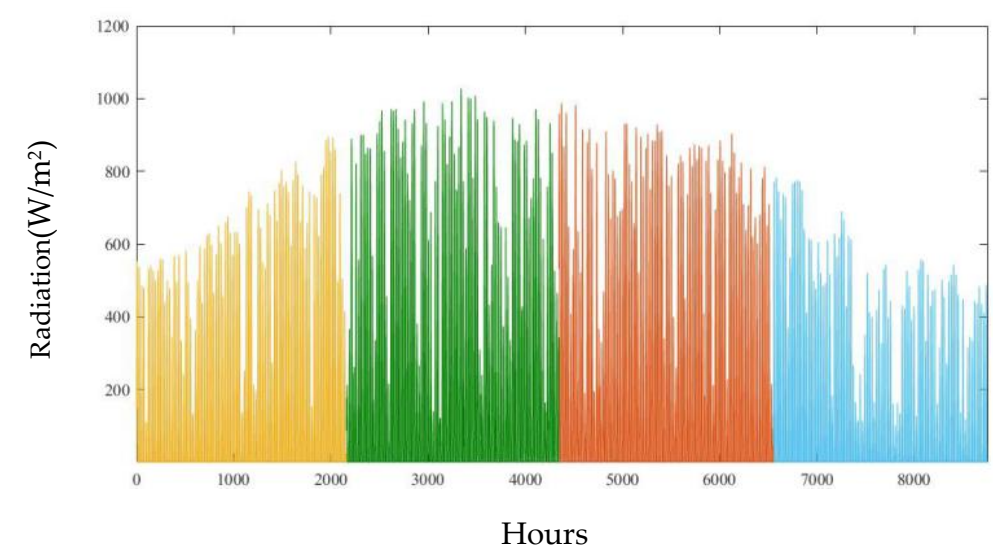

Figure 14. Correcting the annual solar radiation according to the route.

\subsection{Experimental Results}

The operating environment here was $1200 \mathrm{~W} / \mathrm{m}^{2}, 25^{\circ} \mathrm{C}$ temperature, and the power generated by the PV system was fluctuate around $270 \mathrm{~kW}$. Solar PV power generation systems include solar cell components, hybrid energy storage devices, diesel, controllers, and inverters. Here, the AC bus voltage was $440 \mathrm{~V}$ and the AC frequency was $60 \mathrm{~Hz}$ during the simulation, and the ship's operating conditions were referenced from being anchored. The basic diagram of the solar photovoltaic power generation system is shown as Figure 15. 


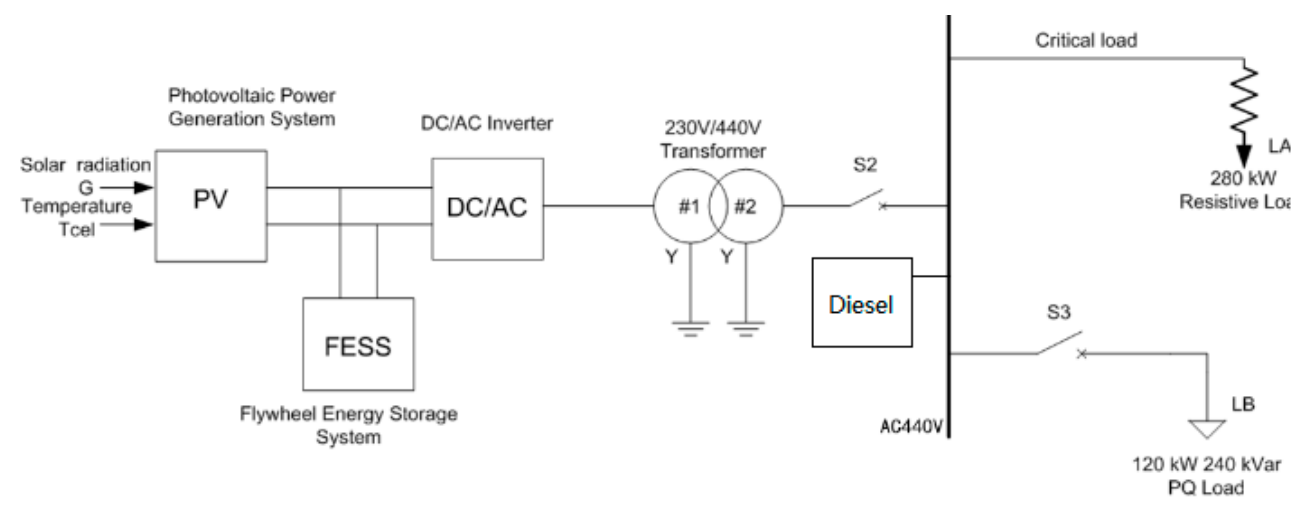

Figure 15. Photovoltaic power generation system.

After the route and time were selected, Equations (29) and (30) of the solar PV power generation system were used to calculate output power.

$$
\begin{gathered}
P_{P V(\mathrm{t})}=\eta_{P V} \times A_{P V} \times I_{t} \\
\eta_{P V}=\eta_{P V_{r} \text { rated }} \cdot \eta_{M P P T}\left[1-\varepsilon\left(T_{c}-T_{c_{\_} r e f}\right)\right]
\end{gathered}
$$

where $I_{t}$ is the light radiation intensity, $\eta_{P V}$ is the conversion efficiency of the solar panel, $A_{P V}$ is the area for laying the photovoltaic panels on the deck, $\eta_{P V_{-} \text {rated }}$ is the rated PV conversion efficiency of the PV module, $\eta_{M P P T}$ is the conversion efficiency under MPPT control, $\varepsilon$ is the power temperature coefficient of the PV module, $T_{\mathcal{C}}$ is the temperature of the surface of the PV module, and $T_{c_{-} \text {ref }}$ is the temperature of the PV module under standard conditions.

The fluctuation of the inclination is intense and random, and the radiation of the photovoltaic panel changes frequently. Figures 16 and 17 show the fluctuation of solar radiation and PV output power in the short-term. The result of the proposed model shows that the actual power output of PV panel fluctuates randomly within a range (250-275 kW), $265 \mathrm{~kW}$ is the average value in one hour. If we adopt the traditional model (fixed PV panel angle) and do not consider the change of inclination as a variable, the power output of Figure 18 can be obtained. Obviously, comparing with the result of our proposed model, the cases of $\delta=4$ and $\delta=8$ in Figure 18 are both an ideal state, the extra energy production are higher and lower than the result of the proposed model as shown in Figure 17 by $5 \mathrm{~kW}$ and $15 \mathrm{~kW}$ respectively. If this error is enlarged to the annual scale, the extra energy production $30,000-60,000 \mathrm{kWh}$ will be mistaken as the value that should be considered in the power planning.

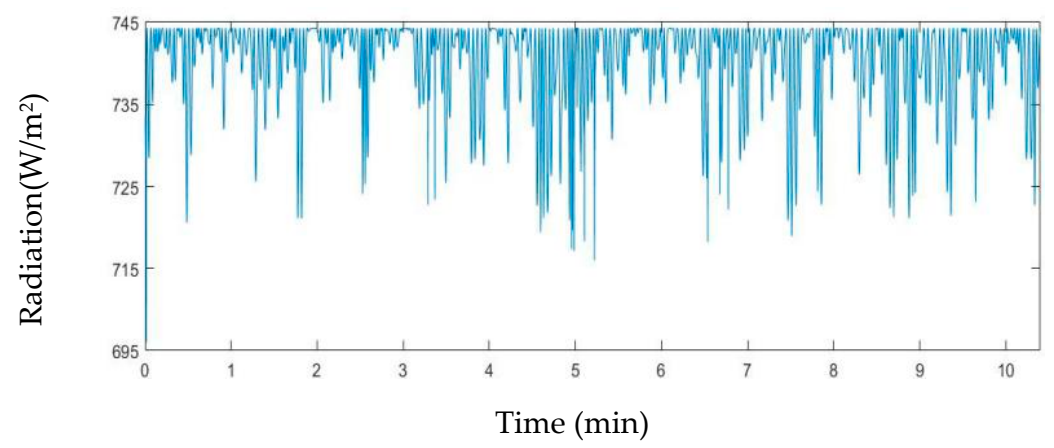

Figure 16. Fluctuation of solar radiation. 


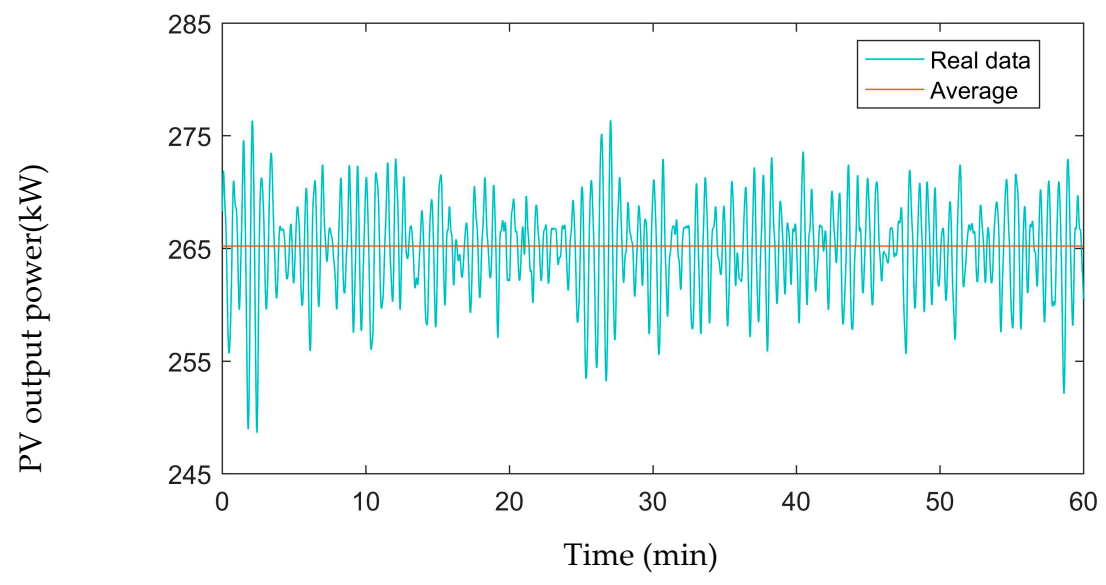

Figure 17. Fluctuation of the photovoltaic system over $1 \mathrm{~h}$.

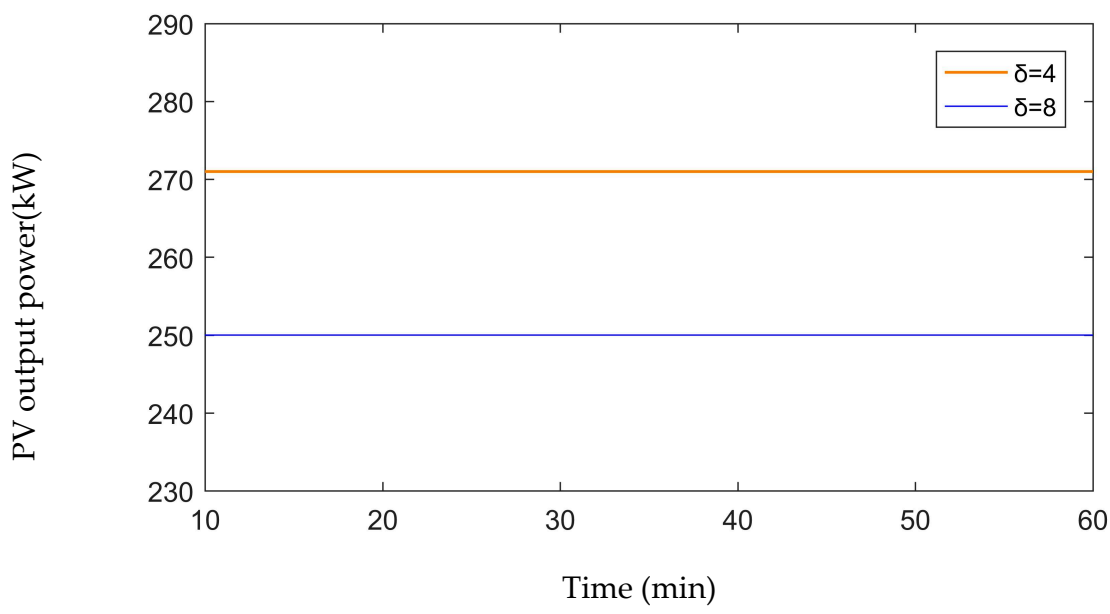

Figure 18. Power generation of the photovoltaic system with a traditional model.

According to the calculation model proposed in previous section, Figures 19 and 20 show the short-term power prediction based on five methods under level-5 and level-3 sea state within $10 \mathrm{~s}$ for a shipborne solar power system, Table 4 shows the MAPE and RMSE value of forecasting the energy production. It can be seen that the power prediction accuracy with MHPSO-LSTM structure is improved by $50 \%$ to $70 \%$ compared with other methods. Therefore, the proposed algorithm reveals better prediction performance in forecasting the short-term power fluctuation.

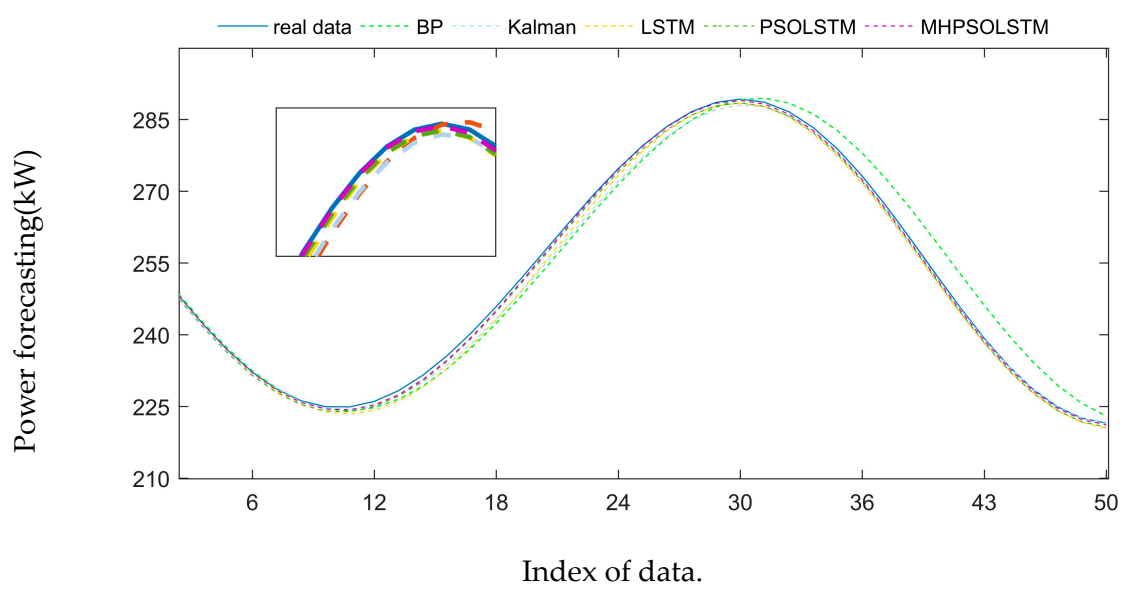

Figure 19. Power fluctuation forecasting under level-5 sea state. 


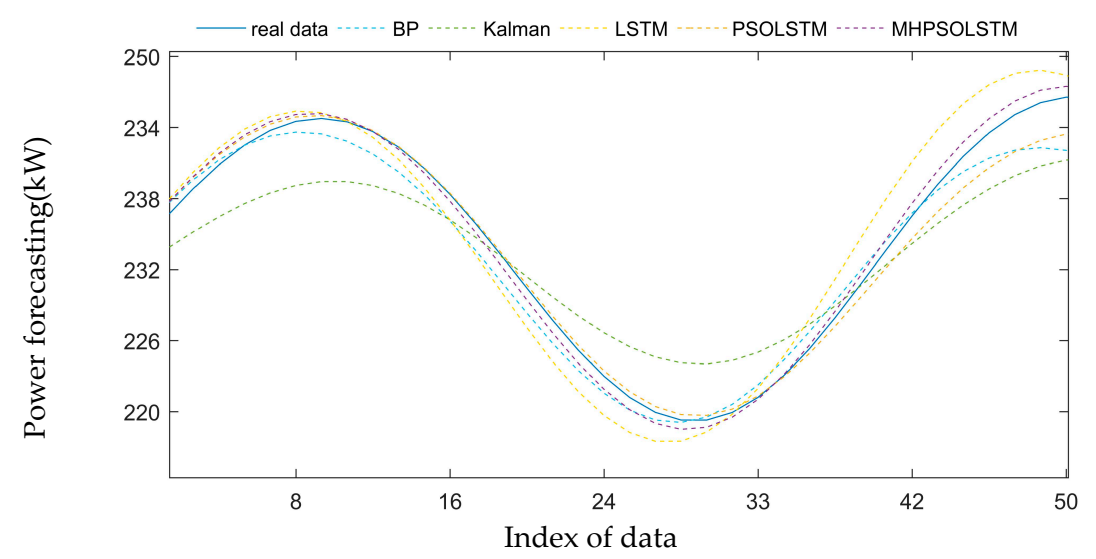

Figure 20. Power fluctuation forecasting under level-3 sea state.

Table 4. The MAPE and RMSE values of the 5 methods for forecasting the energy production.

\begin{tabular}{lllllll}
\hline \multicolumn{2}{c}{ Method } & BP NN & Kalman & LSTM & PSO-LSTM & MHPSO-LSTM \\
\hline \multirow{2}{*}{ Level 3 } & MAPE\% & 16.58 & 15.65 & 12.63 & 12.84 & 6.87 \\
& RMSE & 0.2015 & 0.1921 & 0.1816 & 0.1643 & 0.0982 \\
\hline \multirow{2}{*}{ Level 5 } & MAPE\% & 14.02 & 13.42 & 11.027 & 7.06 & 4.71 \\
& RMSE & 0.0311 & 0.0384 & 0.0353 & 0.0366 & 0.0125 \\
\hline
\end{tabular}

\subsection{Economic and Environment Influence}

Table 5 demonstrate the economic and emissions influence under different calculation model of shipborne PV system. In our cases, generating side including diesel generator and solar PV power generation system, case 1 is the shipborne PV system with traditional model, case 2 representative the PV system under considerate the effect of ship's sway.

Table 5. The cost of marine power grid under two models.

\begin{tabular}{lll}
\hline & Case 1 & Case 2 \\
\hline Fuel cost $(\$)$ & $6,252,155$ & $(6,238,000 ; 6,301,000)$ \\
Dioxide Emissions(kg) & $1,658,220$ & $(1,654,368 ; 1,670,640)$ \\
\hline
\end{tabular}

This paper considers the hourly fuel consumption $F$ of diesel generators, defined as

$$
F=\eta \cdot P_{d}
$$

where the $\eta=0.264(\mathrm{~L} / \mathrm{h})$ is diesel generator fuel consumption coefficient, $P_{d}$ is the power generation of the diesel per hour. The hourly fuel cost defined as

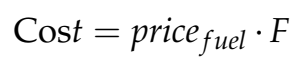

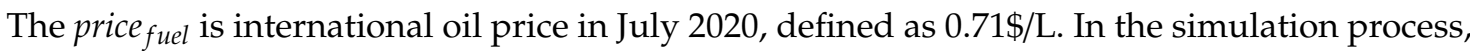
the estimated annual energy production of diesel of ship power stations under different model is $3,975,644 \mathrm{kWh}$ and $(3,969,636-4,001,909) \mathrm{kWh}$ respectively, the capacity of diesel in PV power generation system is $2 \mathrm{MW}$. According to the $\mathrm{CO}_{2}$ emission coefficient, set as $0.92\left(\mathrm{~kg} \mathrm{CO}_{2} / \mathrm{kg}\right)$ in this paper, we also could estimate the amount of dioxide emissions.

Due to the swing of the PV panel, the fuel cost and $\mathrm{CO}_{2}$ emissions of the auxiliary diesel also not a fixed value, but fluctuates within a range, such calculation results could provide a more reliable reference for formulating the energy management strategy of the power grid or the configuration method of energy storage. It is worth noting that the upper limit value of dioxide emissions in case 2 
is $12,420 \mathrm{~kg}$ higher than case 1 , studies related to optimize scheduling that consider environmental protection should take this neglected numerical difference as an important operational constrains. Thus, the proposed calculation model can be used in calculate the power output of shipborne PV, which could provide more reliable operation constraint condition for grid optimization.

\section{Conclusions}

Considering the inaccuracy of traditional marine photovoltaic power calculation models, this paper has proposed an improved mathematical model of PV power calculation via considering the effect of ship movements leading to the change of PV panel inclination. According to a rotation matrix, the roll and pitch are reduced to one angle and then introduced into the radiation calculation formula, where the radiation is corrected according to the latitude, longitude, and season. The proposed mathematical model provides a more realistic model and an appropriate calculation method for shipborne PV systems under different sea conditions, also this could be used as a reference for a series of stability control strategies for the output power of PV panels. Secondly, the MHPSO algorithm was improved for the prediction of PV panel inclination change. By reforming the structure and speed update equation of a PSO algorithm, the capacity of convergence was improved; thereby, increasing the solution speed of the system. Meanwhile, the MHPSO and LSTM models were combined to predict the motion of a PV panel. This structure optimizes the initial weights and thresholds of LSTM in order to overcome the problem of inaccurate parameter selection. In addition, we have used five different models, including MHPSO-LSTM, Kalman filter, LSTM network, BP neural net structure, and a PSO-LSTM algorithm to forecast the movement data of photovoltaic panels under the fifth- and third-level sea states.

Our cases show that the proposed method has higher learning accuracy and better performance prediction than the existing methods. The comparison of grid economics and carbon dioxide emissions also shows the necessity of the proposed model. Finally, the ultrashort-term power prediction of a photovoltaic system has been carried out via predicting the fluctuation of the photovoltaic panel inclination.

In future work, reformation for the MHPSO model will be studied to further raise the prediction precision of shipborne PV panels, and this will be combined with other heuristic algorithms to further study the model performance.

Author Contributions: Conceptualization, L.Z.; methodology, L.Z.; software, B.Z. and L.Z.; validation, X.P.; formal analysis, L.Z.; investigation, L.Z.; resources, L.Z. and B.W.; data curation, L.Z.; writing—original draft preparation, L.Z.; writing-review and editing, X.P.; visualization, B.Z.; supervision, X.P.; project administration, X.P.; and funding acquisition, X.P. All authors have read and agreed to the published version of the manuscript.

Funding: This research was funded by the Ministry of Industry of People's Republic of China (25B01-02) and supported by the Fundamental Research Funds for the Central Universities (3072020CFT2403).

Acknowledgments: The authors would like to acknowledge the project “Energy Management System of MVDC Integrated Electric Propulsion System" of the Basic Product Innovation Research Program for supplying equipment used for experiments

Conflicts of Interest: The authors declare no conflict of interest.

\section{References}

1. Green, M.A.; Dunlop, E.D.; Levi, D.H. Solar cell efficiency tables (version 55). Prog. Photovolt. 2020, 28, 3-15. [CrossRef]

2. Tang, J.; Xiong, B.; Huang, Y.; Yuan, C.; Su, G. Optimal configuration of energy storage system based on frequency hierarchical control in ship power system with solar photovoltaic plant. J. Eng. 2017, 13, 1511-1514. [CrossRef]

3. Wen, S.L.; Wang, Y. Proactive frequency control based on ultra-short-term power fluctuation forecasting for high renewables penetrated power systems. IET Renew. Power Gener. 2019, 13, 2166-2173. [CrossRef]

4. Qiu, Y.C.; Sun, Y.W.; Yuan, C.Q. Review on the Application and Research Progress of Photovoltaics-ship Power System; IEEE: New York, NY, USA, 2015; pp. 523-527. 
5. Buyukozkan, G.; Karabulut, Y.; Mukul, E. A novel renewable energy selection model for United Nations' sustainable development goals. Energy 2018, 165, 290-302. [CrossRef]

6. Martinez-Rubio, A.; Sanz-Adan, F.; Santamaria, J. Optimal design of photovoltaic energy collectors with mutual shading for pre-existing building roofs. Renew. Energy 2015, 78, 666-678. [CrossRef]

7. Dolara, A.; Lazaroiu, G.C.; Leva, S.; Manzolini, G. Experimental investigation of partial shading scenarios on PV (photovoltaic) modules. Energy 2013, 55, 466-475. [CrossRef]

8. Díaz-Dorado, E.; Suárez-García, A.; Carrillo, C.J.; Cidrás, J. Optimal distribution for photovoltaic solar trackers to minimize power losses caused by shadows. Renew. Energy 2011, 36, 1826-1835. [CrossRef]

9. Golestaneh, F.; Pinson, P.; Gooi, H.B. Very short-term nonparametric probabilistic forecasting of renewable energy generation-with application to solar energy. IEEE Trans. Power Syst. 2016, 31, 3850-3863. [CrossRef]

10. Kong, W.C.; Dong, Z.Y.; Jia, Y.W.; Hill, D.J.; Xu, Y.; Zhang, Y. Short-Term Residential Load Forecasting Based on LSTM Recurrent Neural Network. IEEE Trans. Smart Grid 2019, 10, 841-851. [CrossRef]

11. Wang, Y.L.; Soltani, M.; Hussain, D.M.A. Ship attitude prediction based on input delay neural network and measurements of gyroscopes. In Proceedings of the American Control Conference, Seattle, WA, USA, 24-26 May 2017; pp. 4901-4907.

12. Liu, J.; Shahroudy, A.; Xu, D.; Kot, A.C.; Wang, G. Skeleton-Based Action Recognition Using Spatio-Temporal LSTM Network with Trust Gates. IEEE Trans. Pattern Anal. Mach. Intell. 2018, 40, 3007-3021. [CrossRef] [PubMed]

13. Liu, Y.Q.; Shi, J.; Yang, Y.P.; Lee, W.J. Short-Term Wind-Power Prediction Based on Wavelet Transform-Support Vector Machine and Statistic-Characteristics Analysis. IEEE Trans. Ind. Appl. 2012, 48, 1136-1141. [CrossRef]

14. Monteiro, C.; Bessa, R.; Miranda, V.; Botterud, A.; Wang, J.; Conzelmann, G.; INESC. Wind Power Forecasting: State-of-the-Art 2009; Argonne National Lab.: Argonne, IL, USA, 2009; pp. 1-216.

15. Zhou, W.D.; Xing, Z.J.; Bai, W.B.; Deng, C.C.; Xie, Y.E.; Wu, X.D. Route planning algorithm for autonomous underwater vehicles based on the hybrid of particle swarm optimization algorithm and radial basis function. Trans. Inst. Meas. Control. 2019, 41, 942-953. [CrossRef]

16. He, H.; Hu, R.; Zhang, Y.N.; Zhang, Y.; Jiao, R.H. A Power Forecasting Approach for PV Plant based on Irradiance Index and LSTM. In Proceedings of the 2018 37th Chinese Control Conference, Wuhan, China, 25-27 July 2018; Chen, X., Zhao, Q.C., Eds.; IEEE: New York, NY, USA, 2018; pp. 9404-9409.

17. Bui, D.M.; Le, P.D.; Cao, M.T.; Pham, T.T.; Pham, D.A. Accuracy improvement of various short-term load forecasting models by a novel and unified statistical data-filtering method. Int. J. Green Energy 2020, 17, 382-406. [CrossRef]

18. Song, H.H.; Yu, G.X.; Qu, Y.B. Monitoring and forecasting system for ship attitude motion based on extended kalman filtering algorithm. J. Chin. Inert. Technol. 2018, 26, 6-12.

19. Gers, F.A.; Schmidhuber, J.A.; Cummins, F.A. Learning to forget: Continual prediction with LSTM. Neural Comput. 2000, 12, 2451-2471. [CrossRef] [PubMed]

20. Hochreiter, S.; Schmidhuber, J. Long short-term memory. Neural Comput. 1997, 9, 1735-1780. [CrossRef] [PubMed]

21. Guan, T.; Zhuo, F. An improved SA-PSO global maximum power point tracking method of photovoltaic system under partial shading conditions. In Proceedings of the IEEE International Conference on Environment and Electrical Engineering and IEEE Industrial and Commercial Power Systems Europe (EEEIC/I\&CPS Europe), Milan, Italy, 6-9 June 2017; pp. 1-5.

22. Diagne, M.; David, M.; Lauret, P.; Boland, J.; Schmutz, N. Review of solar irradiance forecasting methods and a proposition for small-scale insular grids. Renew. Sust. Energ. Rev. 2013, 27, 65-76. [CrossRef]

23. Akhtar, M.R.; Raza, M.A.; Abid, A.; Usman, M.; Murtaza, Z.; Gelani, H.E. Degradation of PhotoVoltaic (PV) Panel Performance due to Shading Effect- Case of Pakistan. In Proceedings of the 2019 International Conference on Electrical, Communication, and Computer Engineering (ICECCE), Swat, Pakistan, 24-25 July 2019; pp. 1-6.

24. Pope, K.; Naterer, G.F.; Dincer, I.; Tsang, E. Power correlation for vertical axis wind turbines with varying geometries. Int. J. Energy Res. 2011, 35, 423-435. [CrossRef]

25. Sharma, P.; Agarwal, V. Maximum Power Extraction from a Partially Shaded PV Array Using Shunt-Series Compensation. IEEE J. Photovolt. 2014, 4, 1128-1137. [CrossRef]

(C) 2020 by the authors. Licensee MDPI, Basel, Switzerland. This article is an open access article distributed under the terms and conditions of the Creative Commons Attribution (CC BY) license (http://creativecommons.org/licenses/by/4.0/). 